\title{
Nanoscale Chemical Imaging Using Top-Illumination Tip-Enhanced Raman Spectroscopy
}

\author{
J. Stadler, T. Schmid, and R. Zenobi* \\ Department of Chemistry and Applied Biosciences, ETH Zurich, CH-8093 Zurich, Switzerland
}

\begin{abstract}
We present a new top-illumination scheme for tip-enhanced Raman spectroscopy (TERS) in a gap-mode configuration with illumination and detection in a straightforward fashion perpendicular to the sample surface. This illumination focuses the light tightly around the tip end, which effectively diminishes far-field background contributions during TERS measurements. The setup maintains the entire functionality range of both the scanning probe microscopy and the confocal optical microscopy of the setup. For the first time, we show large $(64 \times 64$ up to $200 \times 200$ pixels $)$, high-resolution TERS imaging with full spectral information at every pixel, which is necessary for the chemical identification of sample constituents. With a scanning tunneling microscope tip and feedback, these TERS maps can be recorded with a resolution better than $15 \mathrm{~nm}$ (most likely even less, as discussed with Figure 6). An excellent enhancement ( $\sim 10^{7} \times$, sufficient for detection of few molecules) allows short acquisition times ( $\ll 1$ s/pixel) and reasonably low laser power (in the microwatt regime) yielding spectroscopic images with high pixel numbers in reasonable time $(128 \times 128$ pixels in $<25$ min). To the best of our knowledge, no Raman maps with similar pixel numbers and full spectral information have ever been published.
\end{abstract}

KEYWORDS Tip-enhanced Raman spectroscopy (TERS), gap-mode TERS, enhanced Raman, spectroscopic imaging, top illumination, scanning tunneling microscopy, nanoscale chemical imaging

1 he necessity to gain chemical information on the nanometer scale has steadily increased due to nanometer sized production in industry and increasing general interest in nanometer sized structures. Structural information is nowadays easy to acquire using high-resolution techniques such as atomic force microscopy (AFM), scanning tunneling microscopy (STM), scanning electron microscopy (SEM), or transmission electron microscopy (TEM). Yet these techniques are limited in terms of chemical information and also by the need for vacuum and electron beam stability of the sample. Vibrational spectroscopy offers more insight into the chemical properties of samples showing their molecular or lattice vibrations. Unfortunately optical spectroscopic techniques are limited in their spatial resolution, and, in the case of Raman spectroscopy, by the weak signal strength. Due to optical diffraction, the resolution is limited to roughly half the wavelength of light unless special near-field techniques are used.

In this article we consider a near-field technique invented in 2000, ${ }^{1-4}$ tip-enhanced Raman spectroscopy (TERS), as a tool to access information about the chemical composition on the nanometer scale. Metallic STM ${ }^{5}$ or metalized AFM ${ }^{1,6}$ tips can be used to confine and enhance the electromagnetic field from the illumination laser beam focused to the apex of the tip. The confinement allows spatial resolution down to tens of nanometers and the enhanced field aids to compensate for the intrinsically small cross section of the

* To whom correspondence should be addressed, zenobi@org.chem.ethz.ch Received for review: 07/12/2010

Published on Web: 10/18/2010
Raman process. With additional signal from the sample, much shorter acquisition times and a use of limited laser power are possible, reducing the strain on the sample.

Here we introduce a new optical configuration with an illumination directly from the top and an STM distance control,which allows the use of nontransparent samples while still maintaining a high numerical aperture (NA) and an approximate rotational symmetry of illumination. In comparison to side illumination TERS setups, ${ }^{7-10}$ the high NA of 0.7 reduces the background signals and confines the laser energy more efficiently to the tip. In comparison to epiillumination TERS systems, ${ }^{1,5,11}$ the top illumination/collection system is not limited to transparent samples but allows observation of opaque samples as well.

Until now, two working approaches and one proof of concept have appeared in literature for studying opaque samples by TERS, the side illumination setups mentioned already in refs $7-10$ and an approach based on a parabolic mirror as a focusing element. ${ }^{12-14}$ The parabolic mirror has the advantage of providing a NA up to 1 and is thus superior in terms of focusing capability. However, the alignment of the beam and the parabolic mirror is very delicate and difficult. Slight angular misalignment defocuses the laser beam significantly and renders the system unusable. ${ }^{15}$ Hence, this optical configuration is not widely used: only two research groups (Meixner, Pettinger) have successfully employed this approach for near-field experiments. The only working top illumination setup with lens-based focusing previously described ${ }^{16}$ had a far lower NA and a special higher order laser mode (the so-called "doughnut" mode) in 


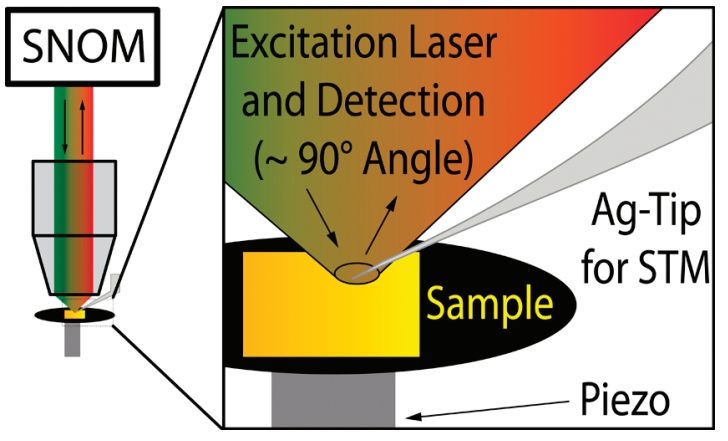

FIGURE 1. Schematic of the instrument (NTMDT, NTEGRA Spectra Upright): top-illumination TERS instrument with $100 \times 0.7 \mathrm{NA}$ objective (Mitutoyo), $100 \times 100 \times 10 \mu \mathrm{m}$ scanning piezo, four way grating turret with $150,600,1800 \mathrm{~L} / \mathrm{mm}$ and echelle grating, EMCCD (Andor, Newton 971), white light illumination, live view CCD, 632.8 $\mathrm{nm} \mathrm{He}-\mathrm{Ne}$ and $532 \mathrm{~nm}$ solid-state laser. Inset: enlarged view of the angled tip on the sample surface and the focused laser beam.

combination with specially designed (resolution limiting) glass fiber tips on a tuning fork in AFM tapping mode feedback. Schultz et al. were able to present near-field signals with a contrast of around 2 and a resolution of $200-300 \mathrm{~nm}$, which may have been limited by the increased background from the small NA used. The low enhancement only allowed determination of the near-field signal after subtraction of a far-field spectrum. We show here that strong near-field enhancement in top illumination can be achieved directly with standard Gaussian mode lasers and standard etched full-metal STM tips in the gap-mode configuration. The term "gap-mode TERS" describes the use of the highly enhanced electromagnetic field, confined in between a metal tip and a metal surface, as an excitation source. This field is enhanced by an induced image dipole in the substrate, ${ }^{17}$ the lightning rod effect, ${ }^{18}$ and surface plasmon resonance. ${ }^{19,20}$ The emitted Raman signal from the sample is enhanced again by the tip and scattered into the far-field for detection. ${ }^{17}$

With this setup, we are able to show full TERS maps with a resolution better than $15 \mathrm{~nm}$ and acquisition times as short as $0.05 \mathrm{~s}$ per pixel. These short acquisition times can be realized based on an enhancement factor of $>10^{7}$ and allow maps of, for example, $128 \times 128$ pixels in less than $25 \mathrm{~min}$.

All experiments in this paper were conducted on a combined STM/Raman spectrometer (NT-MDT, Russia, NTEGRA Spectra Upright, schematic see Figure 1). The instrument consists of a $100 \times 100 \times 10 \mu \mathrm{m}$ piezo scanner for sample movement, controlled by an STM feedback unit, and allows full visibility of tip and sample simultaneously. The tip is held at a $30-50^{\circ}$ angle with respect to the sample surface, can be positioned laterally, and is approached by moving the sample into tunneling contact. The STM tip holder unit has been custom designed to fulfill these needs. From the top, a white light source with a CCD camera offers a magnified live view of the sample. This CCD can be used to monitor the sample, place the tip directly onto sample areas of interest, and coarsely align the laser to the tip. The incoming laser light from a linearly polarized $632.8 \mathrm{~nm}, 15$ $\mathrm{mW} \mathrm{He}-\mathrm{Ne}$ laser is focused by a long working distance objective (Mitutoyo, 100 $\times \mathrm{NA}=0.7$ ) and can be scanned over the sample surface using piezoelectrically driven mirrors. Light emitted or scattered from the sample is collected by the same optics, separated by an edge filter, and directed into a quadruple grating Raman spectrometer (integral part of the NT-MDT system). The photons from the selected part of the spectrum are then acquired by an electron multiplying CCD (EMCCD), which is Peltier cooled to $-90^{\circ} \mathrm{C}$ (Andor, Newton 971).

For vapor coating of metals, a Bal-Tec MED 020 vapor coating chamber was used. Solvents were purchased from various suppliers (incl. Aldrich, Fluka, Acros) in analytical grade purity. As analytes, brilliant cresyl blue (BCB, Fluka) and nile blue A (NB, Fluka) dyes dissolved and diluted in methanol were used.

As a conductive sample support for STM, small squares of freshly cleaved mica were coated with $100 \mathrm{~nm}$ of gold (Bal-Tec, 99,99\%) by resistive heating, using a deposition rate around $0.1 \mathrm{~nm} / \mathrm{s}$ at pressures below $10^{-5} \mathrm{mbar}$. The structure of the gold coating was determined by AFM and STM and showed corrugation of less than $5 \mathrm{~nm}$ over the entire, otherwise homhogeneous surface. Gold surfaces prepared in this way were then spin-coated with a solution of $5 \times 10^{-5} \mathrm{~mol} / \mathrm{L}$ of $\mathrm{BCB}$ or NB in methanol. Any roughness of the gold film can lead to surface-enhanced Raman (SER) activity. However, the Au surfaces did not show any SER activity.

For production of the STM tips, silver wire with a diameter of $0.25 \mathrm{~mm}$ and $99,99 \%$ purity (Aldrich) was sharpened by electrochemical etching. As an etchant, a mixture of $1: 2$ (v/v) perchloric acid (Riedel-de Haën): methanol was prepared. The metal wire was cut to an appropriate length and immersed into the etching solution together with a $1 \mathrm{~mm}$ diameter gold wire loop as a counter electrode. A voltage of $10 \mathrm{~V}$ was applied between the electrodes and switched off by a custom-made circuit ${ }^{21,22}$ for cutting the power. The etching was switched off within $10 \mathrm{~ms}$ upon a steep drop in current, which appears due to the formation of the tip and the loss of the immersed part of the wire. After etching, the tips were rinsed with methanol and checked visually under a Nikon 360x stereo microscope. Tips with a diameter of $<100 \mathrm{~nm}$ could be produced routinely, as shown in Figure 2. This tip etching procedure yields tips that combine a sufficient sharpness and high enhancement with reasonable stability for STM scanning. For the TERS experiments, freshly etched silver tips were used and, unless otherwise stated, less than $15 \mu \mathrm{W}$ of laser power was focused onto the sample.

All Raman spectra were exported in ASCII format without any background correction for evaluation in Origin 8 (Origin Lab Corp., USA), 3D images were exported and evaluated using SPIP 5.0.1 (Image Metrology A/S, Denmark), and all $2 \mathrm{D}$ images were processed in the vendor software directly 


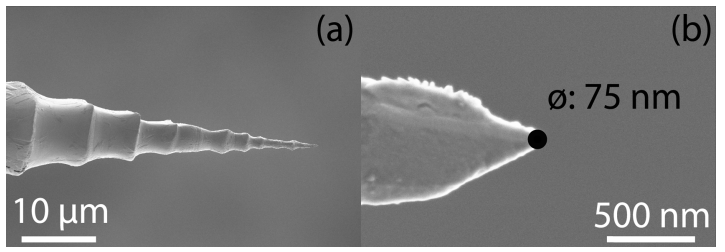

FIGURE 2. SEM image of silver tip showing a radius of curvature at the apex of about $75 \mathrm{~nm}$. (a) Overview showing pencil-like shape of the tip, and (b) tip end; the black circle corresponds to a diameter of $75 \mathrm{~nm}$.

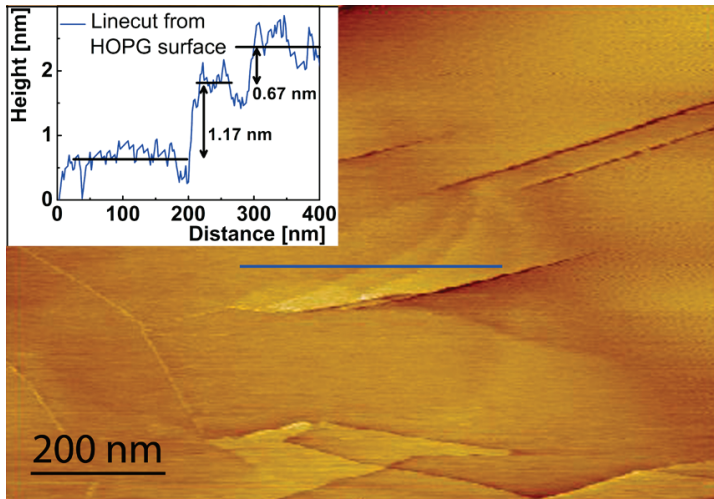

FIGURE 3. $1086 \times 758 \mathrm{~nm}$ STM scan of a flat highly ordered pyrolytic graphite (HOPG) surface with an etched silver tip, $2 \mathrm{~nm}$ step size. The image shows steps of graphite sheets on the HOPG surface with 0.67 and $1.17 \mathrm{~nm}$ height, corresponding to two and four layers of graphite. Graph: line profile over the graphite sheets with a noise level of $0.5 \mathrm{~nm}$ and a lateral resolution of $<10 \mathrm{~nm}$ during scan (linear correction applied).

(NTMDT, Russia). For STM data, a linear line-by-line height correction was usually applied to compensate for drift during acquisition.

To determine the quality of the STM imaging, a sample of highly ordered pyrolitic graphite (HOPG) was scanned at $2 \mathrm{~nm} /$ pixel resolution (see Figure 3). The scan shows steps with heights of 0.67 and $1.17 \mathrm{~nm}$ closely matching two to three layers of graphite (the height of freshly exfoliated graphite layers is $0.335 \mathrm{~nm}^{23}$ ), which are clearly resolved. The line profile also documents an average roughness $<0.5$ $\mathrm{nm}$ in the $Z$ direction. Judged by the width of the steps in the line profile, a lateral resolution of $<10 \mathrm{~nm}$ was determined. Consecutive scans showed the reproducibility of the scans and a drift of $2.2 \mathrm{~nm} / \mathrm{min}$ within $2 \mathrm{~h}$. Sample drift strongly depends on the nature of the sample, on the room temperature, and on the type of sample fixation and can thus vary between measurements.

The standard procedure for recording a TERS map involves the following steps: First, the etched tip and the laser have to be aligned. Therefore the tip is placed onto a homogeneous sample area and the laser focus is scanned over the tip using the piezoelectrically driven scanning mirrors. This results in a spectral intensity map from which the best alignment for tip and laser can be determined (see Figure 4). Until present this had to be done manually via trial and error or guided by external microscopes in existing gap-
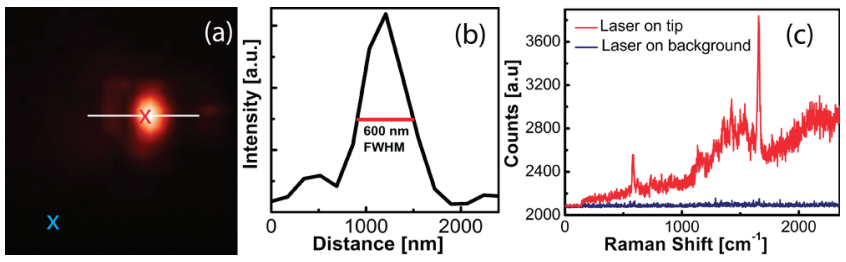

FIGURE 4. (a) $5 \times 5 \mu \mathrm{m}$ Raman map of the area around the tip in contact with a spin-coated sample of $\mathrm{BCB}\left(10 \mu \mathrm{L}, 5 \times 10^{-5} \mathrm{~mol} / \mathrm{L}\right)$ on gold, obtained by scanning the laser over the tip in tunneling contact (tip introduced from the right-hand side). Crosses indicate the locations of spectra reported in (c). (b) Intensity profile along the white line in (a), showing a focus size of $600 \mathrm{~nm}$ in diameter. (c) TERS spectrum taken at the center of the tip on the surface (red) and normal Raman spectrum obtained at an unenhanced area (blue) showing a contrast of $>20 ; 1$ s per spectrum.

mode configurations, with a far greater margin of error and frequent loss of signal. ${ }^{8,24,25}$ With this mapping mode, a quick, easy, and reliable alignment is possible. Figure $4 \mathrm{a}$ illustrates this procedure: it shows enhancement by the tip while illuminated by the confocal laser, a slightly elongated circular shape (the tip was introduced from the right-hand side in this image), and (b) the spot size of the focused laser of $\sim 600 \mathrm{~nm}$ in diameter. The laser focus is aligned to the center of this spot (indicated by a red cross in Figure 4a), and the sample is subsequently scanned underneath the fixed tip and laser position. During this scan, a complete Raman spectrum is acquired at every pixel. These spectra are saved as a $4 \mathrm{D}$ data set consisting of $x, y$ position, spectral shift, and the Raman intensities. This 4D map can then be reviewed spectrum by spectrum, or intensity maps of certain marker bands can be displayed revealing typical signatures of different chemicals.

When Raman maps are recorded, additional information is obtained, such as the topography from the piezo scanner, the error signals from the STM feedback, and the Rayleigh scattering intensity of the surface. The combination of a Raman intensity map and topography information is shown in Figure 5. It can be used to correlate the topography and the chemical composition of the sample with high lateral resolution. The map in Figure 5 was acquired on a $45 \mathrm{~nm}$ thick gold film, spin-coated with $5 \mu \mathrm{L}$ of $5 \times 10^{-4} \mathrm{~mol} / \mathrm{L}$ nile blue together with $5 \mu \mathrm{L}$ of $5 \times 10^{-5} \mathrm{~mol} / \mathrm{L}$ of $\mathrm{BCB}$. Due to the similar Raman cross sections of these two compounds, the spectra mainly show the signals from the more concentrated nile blue. Figure 5a shows the topography from the STM feedback during acquisition, Figure $5 \mathrm{c}$ shows the Raman intensity of the band at $590 \mathrm{~cm}^{-1}$, and Figure $5 \mathrm{~b}$ is an overlay of the STM height (orange) and the Raman intensity in green. The scan direction in the map was from top left to bottom right. The areas of increased Raman intensity form coherent patterns within several lines of the scan; hence they cannot be attributed to random or shortterm fluctuations of the signal. The Raman intensity on the surface appears homogeneous in a confocal Raman scan, but when increasing the resolution using the TERS tip, we observed an increased Raman intensity of analytes inside 

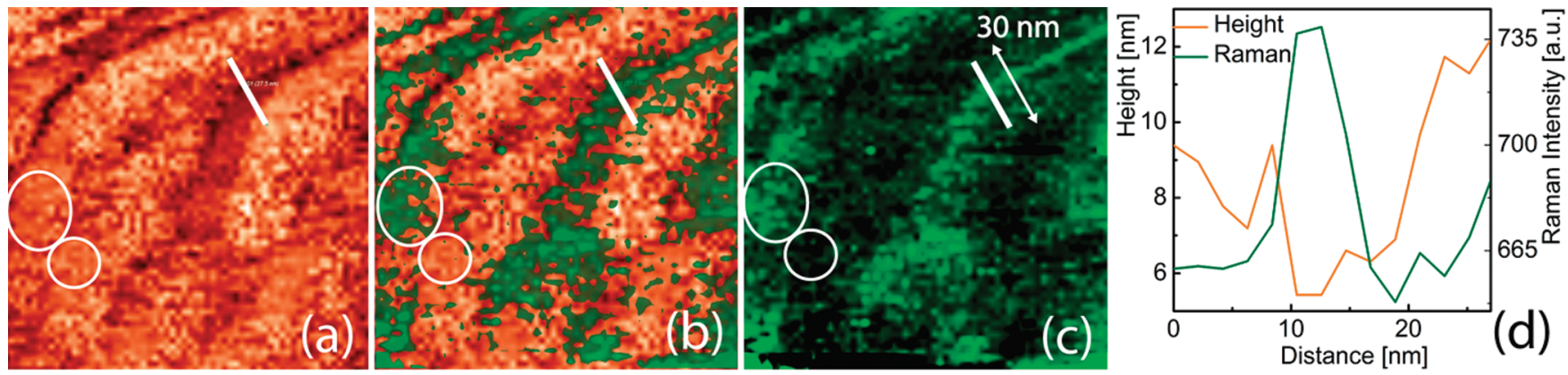

FIGURE 5. TERS map of a $128 \times 128 \mathrm{~nm}$ area of a spin-coated $10 \mu \mathrm{L}$ sample of $5 \times 10^{-5} \mathrm{~mol} / \mathrm{L} \mathrm{BCB}$ and $5 \times 10^{-4} \mathrm{~mol} / \mathrm{L} \mathrm{NB}$ on gold, $64 \times 64$ pixels, 0.05 s per point. (a) STM image obtained during the scan. (b) Overlay of Raman intensity at $580-600 \mathrm{~cm}^{-1}$ (green, translucent) on the height signal (red). (c) Intensity of the $590 \mathrm{~cm}^{-1}$ marker band of nile blue. (d) Height and intensity profile along the white line shown in (a) - (c) showing higher Raman intensity in a trench; white circles show areas of high and low Raman intensity without obvious topographic difference, ruling out distance fluctuations as origin of the intensity changes.

small trenches (2-3 $\mathrm{nm}$ depth) of the gold surface. The $z$-distance dependence of the Raman signal is very strong due to the strong localization of the electromagnetic field to the tip end and the coupling of tip and sample. Experiments and theoretical calculations have shown gap-mode enhancement to decay strongly within $5 \mathrm{~nm}$ and vanish within 15 $\mathrm{nm} \cdot{ }^{26-29}$ Thus, the increased signals could at first glance be attributed to a movement of the TERS tip in the $z$ direction, i.e., due to to an improper STM feedback. However, in the circled regions, two topographically flat regions exhibit a strong difference in the Raman signal, contradicting that the intensity changes arise from a change in tunneling distance. Therefore, we attribute the intensity changes to concentration differences. In other words, not only nanoscale chemical identification but also quantitative information is available from these maps. The graph in Figure $5 \mathrm{~d}$ shows line profiles of the topography and the Raman intensity at the same position (indicated by the white line in Figure $5 a-c$ ), clearly demonstrating two things: first, the highest intensity of the Raman signal is located directly over the shallow trench in the surface and, second, the resolution of both STM image and Raman map in this measurement is well below $15 \mathrm{~nm}$. When this resolution is compared to the size of the STM tip (around $75 \mathrm{~nm}$ in diameter), the field enhancement in the gap is confined to only a fraction of the tip apex, clearly confirming its near-field origin (similar observations have been made experimentally from Hartschuh et al. ${ }^{30}$ as well as calculations by Roth et al. ${ }^{20}$ support this).

Not only the resolution but also the time required to record a TERS map is important, as the stability of the system (drift of sample, tip and laser alignment) limits the acquisition time. The TERS map in Figure 5 consists of 64 $\times 64$ pixels with a $2 \mathrm{~nm}$ step size (a $200 \times 200$ pixel image with $2 \mathrm{~nm}$ step size is shown in the Supporting Information). The step size in nanometers per pixel is not related to the optical resolution of the image but only indicates the traveling distance of the tip in between two recorded spectra. It is only limited by the positioning ability of the scanning piezos (well below $1 \mathrm{~nm}$ per pixel could be used). Both TERS maps were recorded with an acquisition time of $0.05 \mathrm{~s}$ per spectrum, showing that with a sufficiently "hot" tip, short map times and high-resolution Raman maps can be achieved. The overall time for a $128 \times 128$ pixel map with an integration time of $0.05 \mathrm{~s}$ is less than $25 \mathrm{~min}$. Due to the much longer time needed, previous tip-enhanced Raman maps reported in the literature were limited in pixel number and, often, by tedious procedures of acquiring single spectra with subsequent manual repositioning of the tip $(>6 \mathrm{~h}$ for $20 \times 20$ pixels, ${ }^{31}>10$ min for $16 \times 16$ pixels, ${ }^{32}>22 \mathrm{~h}$ for $64 \times 64$ pixels $^{33}$ ).

An additional benefit from a highly enhancing tip is that the thermal strain of the sample can be limited by using low laser power. The laser power used in the majority of the experiments was as low as $15 \mu \mathrm{W}$. Similar results could even be produced using only $4 \mu \mathrm{W}$ of power, albeit at the cost of a longer integration time ( $0.5 \mathrm{~s}$ per spectrum). This shows that we can choose, depending on the type of sample and the information needed, an appropriate balance between acquisition time, spatial resolution, and laser power.

During a series of measurements, targeted to map concentration differences of multiple dyes on a flat surface, consecutively or simultaneously spin-coated with diluted solutions of $\mathrm{NB}$ and $\mathrm{BCB}$, temporal intensity fluctuations of the two dye signals were observed. High-resolution TERS maps were recorded to investigate fluctuations of the ratio of the $\mathrm{NB}$ and $\mathrm{BCB}$ bands. In consecutive spectra within a single line and even while continuously monitoring the TERS signal from a parked tip, strong fluctuations could be observed. Figure 6 shows four spectra taken during a TERS map of a gold surface spin coated with $\mathrm{NB}$ and $\mathrm{BCB}$. The spectra all show the typical bands of $\mathrm{BCB}$ (marker bands at 580 and $1667 \mathrm{~cm}^{-1}$ ) and NB (marker bands at 590 and 1643 $\mathrm{cm}^{-1}$ ). The spectra originate from locations separated by 2 $\mathrm{nm}$ (which is below the previously observed resolution limit), such that only minimal changes in intensity were expected. A drift larger than that can be ruled out due to the short time interval $(0.2 \mathrm{~s})$ between the acquisition of the spectra (less than $1 \mathrm{~s}$ passed during the acquisition of all four spectra). Nonetheless the band intensities of the $580 \mathrm{~cm}^{-1}: 590 \mathrm{~cm}^{-1}$ marker bands changed from 400:30 counts (red spectrum) 


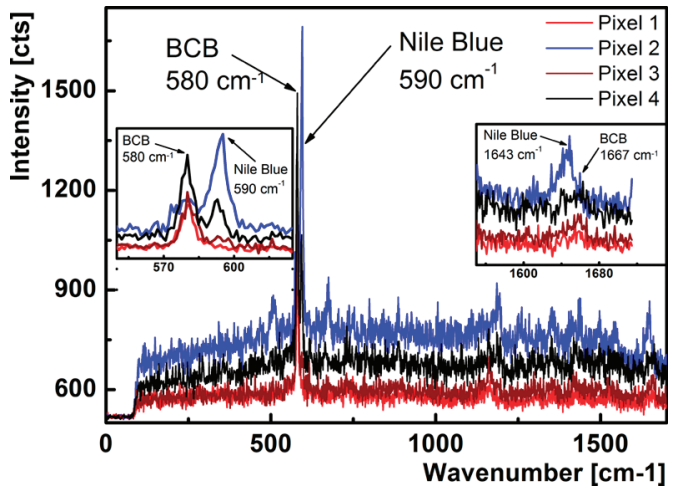

FIGURE 6. Four consecutive TERS spectra (adjacent pixels) from a Raman map of NB and BCB on gold (taken within $1 \mathrm{~s}$ ), showing strong changes in the intensity of the $\mathrm{BCB}$ and NB marker bands at 580 and $1650 \mathrm{~cm}^{-1}$. Left inset shows fluctuating intensities of the main bands: Pixels 1, 2, 3, and 4 yield 400:30, 160:800,500:70, and 700:330 counts, respectively. This behavior can be explained by an extremely localized field probing only few single molecules. $\lambda=$ $632.8 \mathrm{~nm}, 15 \mu \mathrm{W}, 0.1 \mathrm{~s} /$ pixel integration time, taken from a Raman map with $2 \mathrm{~nm}$ step size and $128 \times 128$ pixels.

to $160: 800$ counts (blue) to 500:70 (brown) counts and ended up with an intermediate ratio of 700:330 within less than a second. The overall enhancement during this period was fairly stable and cannot account for the large changes in relative intensity of the NB and BCB marker bands. This leads to the conclusion that the identity of the dye molecules within the enhanced area must have changed during the experiment. The possible causes for this behavior are diffusion of molecules on the surface due to laser heating or the presence of only a few single molecules in an extremely small "hot zone" under the tip apex. The latter could be due to either a low surface coverage of dye (unlikely, as we estimated a significantly higher coverage) or, more likely, an even stronger confinement of the field than the $15 \mathrm{~nm}$ spatial resolution determined from Figure 5. This would automatically lead to a low number of molecules contributing to the Raman signal and also imply an even higher resolution and higher enhancement which is necessary to see a few single molecules. This interpretation appears to be reasonable: single molecule sensitivity for tip-enhanced Raman spectroscopy in gap-mode configuration has already been presented in the literature, ${ }^{27,24,34}$ with high enhancement factors of $10^{6}-10^{8}$.

To estimate a lower boundary for the field enhancement achieved with this setup, we calculate the enhancement factor using eq 1 .

$$
\mathrm{EF}=\frac{\mathrm{I}_{\mathrm{NF}}}{\mathrm{I}_{\mathrm{FF}}} \frac{\mathrm{A}_{\mathrm{FF}}}{\mathrm{A}_{\mathrm{NF}}}
$$

This approach considers the fraction of intensity from the far field $\left(I_{\mathrm{FF}}\right)$ and the near field $\left(I_{\mathrm{NF}}\right)$ from a single vibrational band (the so-called spectroscopic contrast) and divides it by

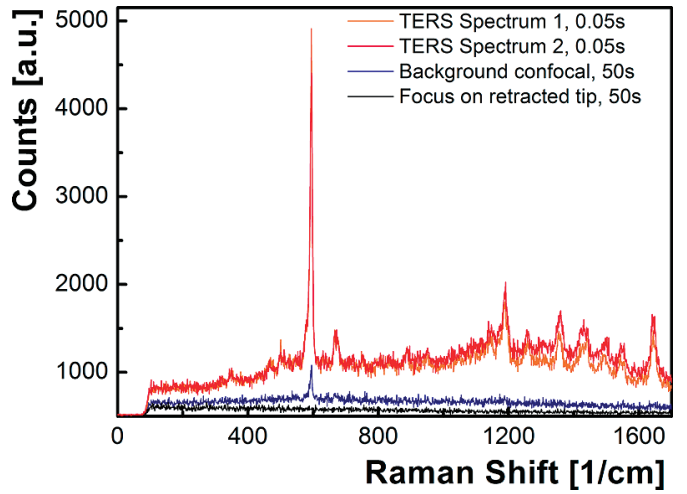

FIGURE 7. Raman spectra from a Raman map of NB on a flat gold surface: (red/orange) two random spectra, $0.05 \mathrm{~s}$ each on tip showing the spectral similarity within the maps; (blue) 50 s spectrum from the sample (tip retracted) to calculate the confocal Raman signal; (black) $50 \mathrm{~s}$ spectrum with the illumination laser focused on the tip end (tip retracted, after measurement) to check the cleanliness of tip after the measurement.

the areas of origin $\left(A_{\mathrm{FF}}, A_{\mathrm{NF}}\right)$, thus representing a signal intensity independent of the number of molecules probed. $I_{\mathrm{FF}}$ and $I_{\mathrm{NF}}$ can be determined from spectra taken with the tip engaged and retracted (Figure 7) after normalization to counts per second. The resolution of the confocal far-field measurement can be deduced from the line profile in Figure $4 \mathrm{~b}$ as the tip will emit a strong Raman signal as long as it is irradiated by the laser spot. The near-field resolution can be determined from the smallest resolved features (appearing in several lines) in the inset of Figure 5.

The strongest Raman signals (which are often chosen for evaluation) were around 21000 counts in 0.05 s. However, we consider it more correct for the calculations to use the averaged near-field intensity over an entire Raman map, amounting to $\sim 2000$ counts in $0.05 \mathrm{~s}$. The area of the Raman map $(512 \times 512 \mathrm{~nm})$ is very similar to the area probed by the confocal laser beam $(600 \times 600 \mathrm{~nm})$, which thus probes a similar number of molecules. In Figue 7 , spectra 1-2 were arbitrarily chosen from the map to show the spectral similarity and only slightly fluctuating enhancement throughout the map. The far-field contribution (Figue 7 , blue spectrum, taken at the position of the blue cross in Figure 4a) yielded 350 counts in $5 \mathrm{~s}$. To check the cleanliness of the tip, a 50 s spectrum from the retracted tip apex was recorded after the scan and is shown in spectrum 4 . With a radius of the confocal spot of $300 \mathrm{~nm}$, and a near-field diameter of $15 \mathrm{~nm}$, which are both conservative estimates, we can calculate an average enhancement factor of $9 \times 10^{6}$ over the entire TERS map. Consideration of the strongest measured spectrum with 21000 counts in $0.05 \mathrm{~s}$ would have yielded enhancements of $\sim 10^{8}$, yet the approach chosen seems more correct to us because the far-field spectrum also represents an average over this entire area. This difference shows that for the calculation of enhancement factors, the sample has to be selected very carefully; concentration differences on the sample can lead to strong overestimation of the enhancement. 
To the best of our knowledge, no full spectral maps with similarly high pixel numbers have been presented in the literature. All previously presented Raman maps either were of much lower pixel number ${ }^{32,35-38}$ or were from selected spectral bands only. ${ }^{16,39,40}$ The work herein represents a very important step for tip-enhanced Raman spectroscopy, allowing the exact localization of substances due to their spectroscopic signature and concentration differences from their signal strength.

Top illumination in TERS offers a series of advantages and can in most cases compete with or outperform the results from known instruments. It combines the advantages of side and bottom illumination TERS instruments and is only limited by the need to work with conductive samples at the moment (STM feedback). An enhancement of $\sim 10^{7}$ (conservative estimate) is in line with the highest reported enhancement factors from the literature which range from $10^{5}$ to $10^{7} \cdot{ }^{35,41}$ At this point it is necessary to mention that due to the different approaches of determining the enhancement factor, these values are not perfectly comparable and should at some point be redetermined in a standardized way. ${ }^{42}$

The resolution of our instrument was determined to be better than $15 \mathrm{~nm}$, which is also a conservative estimate as we took the full width of the smallest features in the measurements. Other authors use the step height or the full width at half-maximum, which would yield about half of this size. Still, our value compares well to existing resolutions in the literature which are on the order of 15-20 nm. ${ }^{35,37,43}$ Recently a value of $4 \mathrm{~nm}$ was published by Kawata et al. ${ }^{44}$ However, in this special case, the resolution does not result directly from the illumination but is the resolution of an induced band shift due to pressure exerted by the AFM tip. This limits their approach to molecules prone to exhibit a band shift upon exerted pressure and have limited choice of marker bands.

Most systems using gap-mode TERS achieve a high enhancement but use a side illumination approach with illumination from a 60 to $70^{\circ}$ angle with long working distance objectives with a NA in the range of $0.3-0.5 .{ }^{45,46}$ Due to the flat illumination geometry and the low NA, the far-field illumination region is large and the laser energy is less localized. The less localized intensity leads to higher background in the spectra and requires higher laser powers. This can lead to bleaching of the sample around the tip or photochemical degradation of the sample. The configuration presented here, with a circular top illumination not only reduces the laser spot size for less background but also confines the laser energy far more efficiently for tip excitation. Additionally, it later on offers the possibility to switch from a Gaussian mode laser beam profile to the so-called doughnut modes for a strongly localized on-axis polarization component which could help excite the tip surface plasmons more efficiently.
Our system offers the full features of confocal laser scanning Raman microscopy, confocal Raman spectroscopy, and back reflection laser intensity measurements. It allows a direct view of the sample for rapid identification of larger sample features and simpler laser alignment.

To conclude, we could show that it is possible to do gapmode TERS imaging with silver tips on a gold surface using an STM feedback in a top illumination and top collection configuration. This setup achieves a resolution of $\leq 15 \mathrm{~nm}$ both in STM and Raman mode and the enhancement factors compare to the best ones shown in literature. The necessary laser intensity has often been neglected until now, but here we can show the use of very low laser powers, in the low microwatt range, for the acquisition of clear Raman spectra in split seconds. This allows for the collection of highresolution Raman maps with the full spectral information in every pixel for chemical identification. We expect this to have strong influence on studies in the field of carbon nanotubes on metal, catalysis, molecular electronics, or selfassembly on metal surfaces.

Acknowledgment. The authors thank I. Olliges-Stadler for the SEM measurements and the Roche Research Foundation for funding.

Supporting Information Available. $200 \times 200$ pixel TERS map of a spin-coated sample of brilliant cresyl blue and nile blue on gold. This material is available free of charge via the Internet at http://pubs.acs.org.

\section{REFERENCES AND NOTES}

(1) Stockle, R. M.; Suh, Y. D.; Deckert, V.; Zenobi, R. Chem. Phys. Lett. 2000, 318, 131-136.

(2) Anderson, M. S. Appl. Phys. Lett. 2000, 76, 3130-3132.

(3) Hayazawa, N.; Inouye, Y.; Sekkat, Z.; Kawata, S. Opt. Commun. 2000, 183, 333-336.

(4) Pettinger, B.; Picardi, G.; Schuster, R.; Ertl, G. Electrochemistry 2000, 68, 942-949.

(5) Pettinger, B.; Picardi, G.; Schuster, R.; Ertl, G. Single Mol. 2002, 3, 285-294.

(6) Hayazawa, N.; Inouye, Y.; Sekkat, Z.; Kawata, S. Chem. Phys. Lett. 2001, 335, 369-374.

(7) Zhang, W. H.; Schmid, T.; Yeo, B. S.; Zenobi, R. Isr. J. Chem. 2007, 47, $177-184$

(8) Pettinger, B.; Ren, B.; Picardi, G.; Schuster, R.; Ertl, G. J. Raman Spectrosc. 2005, 36, 541-550.

(9) Mehtani, D.; Lee, N.; Hartschuh, R. D.; Kisliuk, A.; Foster, M. D.; Sokolov, A. P.; Maguire, J. F. J. Raman Spectrosc. 2005, 36, $1068-$ 1075 .

(10) Hayazawa, N.; Motohashi, M.; Saito, Y.; Ishitobi, H.; Ono, A.; Ichimura, T.; Verma, P.; Kawata, S. J. Raman Spectrosc. 2007, 38, 684-696.

(11) Pettinger, B.; Picardi, G.; Schuster, R.; Ertl, G.J. Electroanal. Chem. 2003, 554, 293-299.

(12) Sackrow, M.; Stanciu, C.; Lieb, M. A.; Meixner, A. J. ChemPhysChem 2008, 9, 316-320.

(13) Steidtner, J.; Pettinger, B. Rev. Sci. Instrum. 2007, 78, 103104.

(14) Stanciu, C.; Sackrow, M.; Meixner, A.J. Microsc. 2008, 229, 247 253.

(15) Drechsler, A.; Lieb, M.; Debus, C.; Meixner, A.; Tarrach, G. Opt. Express 2001, 9, 637-644.

(16) Schultz, Z. D.; Stranick, S. J.; Levin, I. W. Appl. Spectrosc. 2008, 62, $1173-1179$. 
(17) Geshev, P. I.; Dickmann, K. J. Opt. A: Pure Appl. Opt. 2006, 8 S161-S173.

(18) Novotny, L.; Bian, R. X.; Xie, X. S. Phys. Rev. Lett. 1997, 79, 645648.

(19) Demming, A. L.; Festy, F.; Richards, D. J. Chem. Phys. 2005, 122, 7.

(20) Roth, R. M.; Panoiu, N. C.; Adams, M. M.; Osgood, R. M.; Neacsu, C. C.; Raschke, M. B. Opt. Express 2006, 14, $2921-2931$.

(21) Ibe, J. P.; Bey, P. P., J.; Brandow, S. L.; Brizzolara, R. A.; Burnham, N. A.; DiLella, D. P.; Lee, K. P.; Marrian, C. R. K.; Colton, R. J. J. Vacuum Sci. Technol., A 1990, 8, 3570-3575.

(22) Zhang, W. H.; Cui, X. D.; Yeo, B. S.; Schmid, T.; Hafner, C.; Zenobi, R. Nano Lett. 2007, 7, 1401-1405.

(23) Bacon, G. Acta Crystallogr. 1951, 4, 558-561

(24) Zhang, W. H.; Yeo, B. S.; Schmid, T.; Zenobi, R. J. Phys. Chem. C 2007, 111, 1733-1738.

(25) Saito, Y.; Motohashi, M.; Hayazawa, N.; Iyoki, M.; Kawata, S. Appl. Phys. Lett. 2006, 88, 143109.

(26) Raschke, M. B.; Lienau, C. Appl. Phys. Lett. 2003, 83, 5089-5091.

(27) Neacsu, C. C.; Dreyer, J.; Behr, N.; Raschke, M. B. Phys. Rev. B 2006, 73, 4

(28) Domke, K. F. Ph.D. thesis, 2006

(29) Pettinger, B.; Domke, K. F.; Zhang, D.; Schuster, R.; Ertl, G. Phys. Rev. B 2007, 76, 113409.

(30) Hartschuh, A.; Sanchez, E. J.; Xie, X. S.; Novotny, L. Phys. Rev. Lett. 2003, 90, 4.

(31) Deckert, V.; Zeisel, D.; Zenobi, R.; Vo-Dinh, T. Anal. Chem. 1998, 70, 2646-2650

(32) Zhang, D.; Wang, X.; Braun, K.; Egelhaaf, H. J.; Fleischer, M.; Hennemann, L.; Hintz, H.; Stanciu, C.; Brabec, C. J.; Kern, D. P.; Meixner, A. J.J. Raman Spectrosc. 2009, 40, 1371-1376.
(33) Saito, Y.; Verma, P.; Masui, K.; Inouye, Y.; Kawata, S. J. Raman Spectrosc. 2009

(34) Domke, K. F.; Zhang, D.; Pettinger, B. J. Am. Chem. Soc. 2006, 128, $14721-14727$.

(35) Steidtner, J.; Pettinger, B. Phys. Rev. Lett. 2008, 100, 236101

(36) Picardi, G.; Chaigneau, M.; Ossikovski, R.; Licitra, C.; Delapierre, G. I. Raman Spectrosc. 2009, 40, 1407-1412.

(37) Hartschuh, A.; Qian, H.; Georgi, C.; Bohmler, M.; Novotny, L. Anal. Bioanal. Chem. 2009, 394, 1787-1795.

(38) Wang, X.; Zhang, D.; Braun, K.; Egelhaaf, H. J.; Brabec, C. J.; Meixner, A. J. Adv. Funct. Mater. 2010, 20, 492-499.

(39) Saito, Y.; Motohashi, M.; Hayazawa, N.; Kawata, S. J. Microsc. (Oxford, U.K.) 2008, 229, 217-222

(40) Hoffmann, G. G.; de With, G.; Loos, J. Macromol. Symp. 2008, 265, $1-11,312$

(41) Liu, Z.; Wang, X.; Dai, K.; Jin, S.; Zeng, Z.-C.; Zhuang, M.-D.; Yang, Z.-L.; Wu, D.-Y.; Ren, B.; Tian, Z.-Q. J. Raman Spectrosc. 2009, 40, 1400-1406

(42) Schmid, T.; Yeo, B. S.; Zhang, W.; Zenobi, R. Use of tip-enhanced vibrational spectroscopy for analytical applications in chemistry, biology, and materials science. In Tip Enhancement; Satoshi, K., Vladimir, M. S., Eds.; Elsevier: Amsterdam, 2007; pp 115-155.

(43) Anderson, N.; Hartschuh, A.; Novotny, L. Nano Lett. 2007, 7, 577 582.

(44) Yano, T.; Verma, P.; Saito, Y.; Ichimura, T.; Kawata, S. Nat. Photonics 2009, 3, 473-477.

(45) Tarun, A.; Hayazawa, N.; Motohashi, M.; Kawata, S. Rev. Sci. Instrum. 2008, 79, 13706

(46) Pettinger, B.; Ren, B.; Picardi, G.; Schuster, R.; Ertl, G. Phys. Rev. Lett. 2004, 92, 4 